Tomás, J-M. y Gutiérrez, M. (2019). Aportaciones de la teoría de la autodeterminación a la predicción de la satisfacción académica en estudiantes universitarios. Revista de Investigación Educativa, 37(2), 471-485.

DOI: http://dx.doi.org/10.6018/rie.37.2.328191

\title{
Aportaciones de la teoría de la autodeterminación a la predicción de la satisfacción académica en estudiantes universitarios
}

\section{Contributions of the self-determination theory in predicting university students' academic satisfaction}

\author{
José-Manuel Tomás* y Melchor Gutiérrez** \\ *Departamento de Metodología de las Ciencias del Comportamiento, Facultad de Psicología, Universidad de Valencia \\ (España) \\ **Departamento de Psicología Evolutiva y de la Educación, Facultad de Psicología, Universidad de Valencia (España)
}

\section{Resumen}

La literatura especializada ofrece evidencias de que en todo el mundo las tasas de deserción universitaria son elevadas, generando inconvenientes para los propios estudiantes, para la institución a la que pertenecen y para la sociedad en general. Los determinantes del abandono de los estudios son diversos, considerando uno de los más importantes la satisfacción de los estudiantes con su entorno educativo. La satisfacción académica de los estudiantes depende en gran medida del clima motivacional del aula y de la satisfacción de las necesidades psicológicas básicas, fundamento de la teoría de la autodeterminación. En el marco teórico de la motivación autodeterminada y de la psicología positiva, el objetivo de este trabajo es analizar la capacidad predictiva del apoyo a la autonomía por los profesores sobre la satisfacción académica de los alumnos, mediado por la satisfacción de las necesidades psicológicas básicas de los estudiantes. Los participantes son 752 estudiantes universitarios dominicanos. Instrumentos: Percepción de Apoyo a la Autonomía por los Profesores, Satisfacción Necesidades de las Psicológicas Básicas de los Estudiantes, y Conectividad Académica. Los datos se analizan a través de dos

Correspondencia: Melchor Gutiérrez, melchor.Gutierrez@uv.es, Departamento de Psicología Evolutiva y de la Educación. Facultad de Psicología. Av. Blasco Ibáñez 21, 46010 Valencia (España) 
Modelos de Ecuaciones Estructurales con variables latentes, uno con mediación total y otro con mediación parcial. Los resultados muestran que el apoyo a la autonomía se relaciona positivamente con la satisfacción de las necesidades psicológicas básicas; las necesidades básicas se relacionan positivamente con la satisfacción académica; y también aparece un efecto positivo y directo del apoyo a la autonomía por los profesores sobre la satisfacción académica de los estudiantes universitarios.

Palabras clave: psicología de la educación; educación superior; motivación; bienestar; República Dominicana.

\section{Abstract}

The specialized literature offers evidence that university dropout rates are high throughout the world, creating problems for the students themselves, for the institution to which they belong, and for society in general. The determining factors of dropout are diverse, considering the student satisfaction with their educational environment as one of the most important ones. There is also evidence that the students' satisfaction with their academic environment depends to a large extent on the classroom motivational climate and the satisfaction of basic psychological needs, main elements of the self-determination theory. In the theoretical framework of self-determined motivation and positive psychology, the objective of this paper is to analyze the predictive capacity of teachers' autonomy support on students' academic satisfaction, mediated by the satisfaction of the students' basic psychological needs. Participants are 752 Dominican university students. Instruments: Perceived Teachers' Autonomy Support, Students' Basic Psychological Needs Scale, and Academic Connectedness Scale. The data has been analyzed through two Structural Equation Models with latent variables, a total mediational model and a partial mediational model. The results show that support for autonomy is positively related to the satisfaction of basic psychological needs; that basic needs are positively related to academic satisfaction; and that there is also a positive and direct effect of autonomy support by teachers on university students' academic satisfaction.

Keywords: educational psychology; higher education; motivation; well-being; Dominican Republic.

\section{Introducción}

Un problema relacionado con la educación superior que preocupa a las autoridades educativas de todo el mundo es la alta tasa de deserción universitaria. Datos del Ministerio de Educación, Cultura y Deporte de España (MECD, 2016) indican que, aproximadamente, uno de cada cinco estudiantes abandonan la universidad en el primer curso. En el caso de República Dominicana, el 39.3\% de los estudiantes abandonan las aulas, según la Oficina Nacional de Estadística (Corcino, 2017). Las razones de la deserción universitaria pueden ser diversas: psicoeducativas, evolutivas, familiares, económicas, institucionales y sociales. Uno de los principales motivos de abandono está relacionado con la universidad, es decir, con su entorno, sus profesores y las dificultades cotidianas que se les presentan a los estudiantes (Arce, Crespo \& Míguez-Álvarez, 2015). Así pues, si los estudiantes se sienten satisfechos con su entorno educativo, cabe pensar que tendrán menor intención de abandonar sus estudios. 
Suhlmann, Sassenberg, Nagengast y Trautwein (2018) destacan que los estudiantes con una alta dignidad de autoconstrucción y que perciben las normas universitarias más como un respaldo a la autonomía que como una imposición, es decir, como mecanismos para ser altamente independientes, muestran un mayor sentimiento de pertenencia a la universidad, y este sentimiento predice de forma positiva la motivación académica y reduce la intención de abandonar los estudios. Es decir, un buen ajuste entre la persona y el ambiente universitario no solo facilita los sentimientos de pertenencia a la institución sino también el bienestar y la motivación, a la vez que evita el desarrollo de intenciones de deserción. En este marco se habla del bienestar como un resultado y de motivación como un precursor de la trayectoria de los estudiantes universitarios.

Un aspecto a destacar es la importancia del bienestar subjetivo, y la satisfacción académica como elemento de ese bienestar, considerando el éxito académico más allá de las calificaciones escolares. Esto forma parte de la vertiente del desarrollo positivo (Seligman \& Csikszentmihalvi, 2000), investigado con mayor frecuencia en niveles adolescentes (López-Cassá, Pérez-Escoda \& Alegre, 2018), pero también aplicable a los estudiantes universitarios (Baker, Dilly, Aupperlee \& Patil, 2003; Chen, 2015).

El grado en que los centros educativos funcionan como entornos psicológicamente sanos es una cuestión clave dentro de la psicología positiva aplicada a los jóvenes. Baker et al. (2003) se refieren a los factores contextuales que contribuyen al ajuste positivo usando un marcador de bienestar como es la satisfacción académica, en el marco ecológico del desarrollo. Estos autores señalan asimismo que la satisfacción académica para los jóvenes es similar a la satisfacción laboral de los adultos, por lo tanto es un aspecto relevante que forma parte de la calidad de vida de los estudiantes.

Urquijo y Extremera (2017) resaltan el interés creciente que ha despertado el grado de satisfacción de los estudiantes universitarios desde que en 1998 la UNESCO fijase su atención en el estudiante y sus necesidades como eje central del proceso de calidad educativa. Esto ha generado diversas investigaciones sobre la satisfacción vital (Huo \& Kong, 2014) y sobre la satisfacción laboral y la académica (Lent, Singley, Sheu, Schmidt \& Schmidt, 2007).

La satisfacción académica se ha definido como una evaluación cognitivo-afectiva de la satisfacción general con las experiencias académicas, y se basa en el trabajo teórico sobre la satisfacción con la vida de los jóvenes, un aspecto del bienestar subjetivo. La investigación contemporánea sobre la satisfacción académica intenta comprender los procesos complejos que explican las percepciones de los estudiantes sobre la calidad de la vida académica, teniendo en cuenta las interrelaciones entre las variables psicológicas y ambientales que influyen en la satisfacción con la vida académica (Huebner, Ash \& Laughlin, 2001). Por ello es importante considerar la satisfacción académica junto con otros índices de bienestar, utilizando modelos multidimensionales que den cuenta de las interacciones sutiles de los estudiantes con sus entornos. Aunque existe consenso entre los investigadores en cuanto a la importancia de la satisfacción de los estudiantes con sus estudios como una faceta del éxito académico, se sabe poco acerca de los determinantes de esta significativa variable de resultado (Wach, Karbach, Ruffing, Brünken \& Spinath, 2016).

Un marco conceptual que ha demostrado su utilidad al estudiar el proceso que lleva a los estudiantes a sentirse satisfechos con su entorno educativo o al abandono 
académico es la teoría de la autodeterminación -TAD- (Deci \& Ryan, 2008; Medellín Lozano, 2010; Shih, 2013). La TAD es un enfoque de la motivación humana que describe las circunstancias sociales bajo las cuales las personas experimentan bienestar y vitalidad. La investigación sobre la TAD ha demostrado la importancia de la satisfacción de tres necesidades psicológicas básicas (autonomía, competencia, relación) para el crecimiento psicológico, el bienestar óptimo y otros resultados positivos de la educación, afirmación fundamentada en una variedad de estudios y contextos culturales (Cordeiro, Paixão, Lens, Lacante \& Sheldon, 2016). Las necesidades de autonomía, competencia y relación son universales entre las personas y aplicables en todos los aspectos de la vida. La autonomía se refiere a la libertad de elección en el comportamiento propio. La competencia implica sentirse eficaz y capaz de dominar el entorno. La relación consiste en sentirse conectado con los demás (Deci \& Ryan, 2008). Cuando estas necesidades no se ven satisfechas, suelen producirse resultados no adaptativos como el estrés psicológico y las intenciones de abandono académico (Sulea, van Beek, Sarbescu, Virga \& Schaufeli, 2015).

Desde la TAD se señala que una variable de los contextos sociales que facilita el desarrollo del bienestar es el apoyo a la autonomía por las figuras de autoridad. Por ello, los profesores pueden jugar un papel importante en la satisfacción de las tres necesidades psicológicas básicas de los estudiantes. Cuando los estudiantes reciben apoyo a su autonomía se favorece la satisfacción de esas necesidades, mientras que si no se brinda tal apoyo, la satisfacción de las necesidades se frustra (Deci \& Ryan, 2008). Pero el hecho de que los profesores adopten un estilo de apoyo a la autonomía no es suficiente; es necesario que los estudiantes perciban que sus profesores les prestan tal apoyo (Hagger et al., 2007).

La TAD se ha utilizado con frecuencia para explicar el comportamiento de los alumnos en los contextos de la educación física y el deporte (e.g., Liu \& Chung, 2016; Ma, Shenk \& Lai, 2016). Liu y Chung (2016) encuentran relaciones positivas entre la percepción de apoyo a la autonomía por los profesores y la satisfacción de las necesidades psicológicas básicas de autonomía, competencia y relación de los estudiantes universitarios en las clases de educación física. También López-Walle, Balaguer, Castillo y Tristán (2012) encuentran una relación positiva entre el apoyo a la autonomía por los entrenadores y la satisfacción de las necesidades básicas en una muestra de deportistas universitarios. A su vez, observan un efecto de mediación parcial de las necesidades básicas entre la percepción de apoyo a la autonomía y el bienestar psicológico de los deportistas. En este mismo sentido, Wu, Lai y Chan (2014) concluyen que la conducta de los entrenadores predice la satisfacción de las necesidades básicas y éstas la motivación intrínseca de los deportistas universitarios.

Pero la TAD también ha ido tomando progresiva relevancia en la interpretación del comportamiento de los estudiantes en el contexto de otras materias académicas (e.g., Evans \& Bonneville-Roussy, 2016; Gillet et al., 2017; Milyavskaya \& Koestner, 2011; Niemiec \& Ryan, 2009; Orsini, Binnie, Wilson \& Villegas, 2018). Por ejemplo Milyavskaya y Koestner (2011) ponen de relieve la importancia de la satisfacción de las necesidades psicológicas básicas en favor de un mayor bienestar subjetivo en múltiples dominios. También Evans y Bonneville-Roussy (2016) encuentran que la satisfacción de las necesidades psicológicas y la motivación autónoma se relacionan 
positivamente con prácticas más frecuentes y de mayor calidad, así como una mayor preferencia por tareas desafiantes, en estudiantes universitarios de música. Los autores de este trabajo destacan el potencial de la TAD para explicar de manera más completa aspectos interesantes e insuficientemente investigados de este entorno, incluido el bienestar del estudiante, la ansiedad, los preparativos para una carrera a largo plazo en la música y las implicaciones pedagógicas. Griffin (2016) sugiere que la incorporación de actividades en el aula que engendran apoyo a la autonomía puede conducir a una mejor percepción sobre la instrucción y mejorar la motivación y el aprendizaje de los alumnos. A través de un estudio longitudinal a lo largo de un semestre, Gillet et al. (2017) encuentran que un incremento en la satisfacción de las necesidades básicas se asocia con niveles más altos de afecto y esfuerzo positivo, mientras que cuando la tendencia de la satisfacción de las necesidades es descendente, se asocia con niveles más bajos de afecto positivo, esfuerzo y logro. Por último, en el trabajo de Orsini et al. (2018) aparecen las necesidades psicológicas básicas como mediadoras entre el clima de aprendizaje con apoyo a la autonomía y la motivación auto-determinada en estudiantes universitarios de la especialidad de odontología.

Tomando como base el anterior marco teórico, el objetivo de este trabajo consiste en analizar la capacidad predictiva del apoyo a la autonomía por los profesores sobre la satisfacción académica, mediado por la satisfacción de las necesidades psicológicas básicas de los estudiantes (figura 1). Partimos de tres hipótesis: (1) que el apoyo a la autonomía por los profesores está positivamente relacionado con la satisfacción de las necesidades psicológicas básicas de los estudiantes; (2) que la satisfacción de las necesidades psicológicas básicas está positivamente relacionada con la satisfacción académica de los estudiantes; y (3) que el apoyo a la autonomía por los profesores está positivamente relacionado con la satisfacción académica de los estudiantes universitarios.

\section{Método}

\section{Participantes}

Los participantes son 752 estudiantes de la Universidad Autónoma de Santo Domingo (UASD). Ésta, es la única universidad pública en República Dominicana y alberga en torno al 50\% de los estudiantes universitarios del país. La selección de los participantes se realizó mediante muestreo por conveniencia, a través de colaboradores, procurando que la muestra obtenida fuese lo más representativa posible de la población a la que pertenece. Los estudiantes seleccionados estaban matriculados en Ciencias de la Educación (19.3\%), Ciencias Sociales (24.8\%), Educación Inicial y Básica (24.7\%), Educación Física (5.6\%), Filosofía y Letras (8.6\%), y Orientación (17.0\%). Sus edades oscilan entre 18 y 50 años. Del total de la muestra, el 21\% son hombres y el 79\% mujeres. En relación con el nivel de estudios, el $12.8 \%$ estudian $1^{\circ}$ curso, el $41.8 \%$ son de $2^{\circ}$ curso, el $27.4 \%$ son de $3^{\circ}$ curso y el $8 \%$ de $4^{\circ}$ curso. Dado que el número de matriculados en estas facultades alcanza cada año una cifra alrededor de los 35000 estudiantes, la tasa de error para la situación más desfavorable de muestreo se situaría en un 3.5\% para el total de la muestra analizada. 


\section{Instrumentos}

Todos los instrumentos han sido respondidos en una escala tipo Likert de cinco anclajes, desde (1) Totalmente en desacuerdo, hasta (5) Totalmente de acuerdo.

- Apoyo a la autonomía por los profesores. Se utilizó una versión del Learning Climate Questionnaire (Jang, Kim \& Reeve, 2012). La escala viene encabezada por la expresión "En mi Facultad"...y está compuesta por seis ítems (e.g., Siento que mis profesores/as me brindan opciones y oportunidades). Jang et al. (2012) refieren fiabilidades de esta escala entre.89 y.93, en diferentes aplicaciones. Sometida esta escala a un Análisis Factorial Confirmatorio (AFC) con los datos de esta muestra, los resultados ofrecen satisfactorios índices de ajuste $\left(c^{2}(9)=88.04, p<.001, C F I=.98\right.$, $\mathrm{TLI}=.97$, RMSEA =.10) lo cual confirma la estructura unidimensional propuesta por sus autores. La fiabilidad correspondiente a los datos de esta muestra es de $\alpha=.87$.

- Necesidades psicológicas básicas. Se utilizó la "Students' Basic Psychological Needs at School Scale", de Tian, Han y Huebner (2014). Esta escala se encuentra encabezada por la expresión: En mi Facultad... y está compuesta por 15 ítems agrupados en tres factores: Autonomía (e.g., Puedo decidir por mí mismo/a cómo hacer las cosas), Competencia (p.ej.: Soy capaz de adquirir conocimientos nuevos), y Relación (p.ej.: Tengo buenos amigos/as). Los coeficientes alfa aportados por Tian et al. (2014) fueron:.85,.77 y.80, respectivamente. Mediante la aplicación de un AFC a los datos de esta muestra, se han obtenido unos índices de ajuste satisfactorios: $\mathrm{C}^{2}(87)=393.53, p<.001, \mathrm{CFI}=.94, \mathrm{TLI}=.93$, RMSEA $=.06$, lo cual confirma que la escala está formada por los tres factores antes señalados. La fiabilidad obtenida con los datos de esta muestra han sido: Autonomía $(\alpha=.68)$, Competencia $(\alpha=.69)$, y Relación $(\alpha=.74)$.

- Satisfacción académica. Para medir la satisfacción de los estudiantes universitarios con su entorno educativo se ha utilizado una traducción y adaptación de la "School Connectedness Scale", de Nearchou, Stogiannidou y Kiosseoglou (2014). Aunque esta escala se creó para ser utilizada en niveles educativos de enseñanza primaria y secundaria, hemos realizado una adaptación de su contenido al contexto universitario. Es una escala unidimensional, compuesta por cinco ítems (e.g., Me siento feliz de estar en esta Facultad). El AFC llevado a cabo con los datos de esta muestra ha aportado satisfactorios índices de ajuste $\left(c^{2}(8)=41.58, p<.001\right.$, CFI $=.99$, TLI $=.98$, RMSEA =.09), lo cual confirma la estructura de un factor propuesta por Nearchou et al. (2014). Así mismo, la fiabilidad obtenida con los datos de esta muestra ha sido de $\alpha=.81$.

\section{Procedimiento}

El primer paso consistió en solicitar los oportunos permisos al Ministerio de Educación de República Dominicana para llevar a cabo la investigación. Una vez obtenidos estos permisos, se contactó con las autoridades de las facultades universitarias seleccionadas para formar parte de la muestra de estudio; se les explicó en qué consistía la investigación y se les solicitó permiso para llevarla a cabo. Finalmente, se informó al alumnado sobre 
el objetivo de la investigación y se les ofreció la posibilidad de participar libremente. Este estudio cumple los requisitos del código ético de la Asociación Americana de Psicología (APA). Los datos se recogieron durante el mes de mayo de 2017, aplicando los instrumentos en horario habitual de clase, dedicando un tiempo a completarlos no superior a 15 minutos.

\section{Análisis de datos}

El diseño de investigación empleado en este estudio es de corte cuantitativo, correlacional, transversal y los datos se han obtenido mediante encuesta. En el análisis de los datos, primero se desarrollaron diversos AFC para comprobar la validez factorial de los instrumentos en la muestra en estudio; seguidamente se analizó la fiabilidad de los instrumentos mediante el coeficiente alfa de Cronbach; también se calcularon las correlaciones bivariadas entre las variables estudiadas; y finalmente se sometieron a verificación dos modelos de ecuaciones estructurales completos con variables latentes, para predecir la satisfacción académica, a partir de la percepción del apoyo a la autonomía por los profesores y de la satisfacción de las necesidades psicológicas básicas de los estudiantes universitarios. Los modelos confirmatorios se estimaron mediante máxima verosimilitud con correcciones robustas de Satorra-Bentler en los errores estándar y estadísticos e índices de ajuste (Finney \& DiStefano, 2006). Para evaluar el ajuste de los modelos se utilizó el CFI, que considera apropiado para aceptar el modelo el valor de.90 o superior; el RMSEA, con valores menores de.08 considerados aceptables para afirmar que un modelo es plausible; y la prueba de chi-cuadrado (Kline, 2016). La estimación de todos los modelos estructurales se ha realizado con el paquete estadístico Mplus 8 (Muthén \& Muthén, 2017). Los dos modelos probados para verificar la mediación de la satisfacción de las necesidades básicas se muestran en la figura 1. El primero de ellos plantea una mediación total ya que todos los efectos del apoyo a la autonomía por el profesorado son indirectos a través de la satisfacción de las necesidades psicológicas básicas. En el segundo, la mediación se asume parcial, y en ella se especifican efectos directos adicionales entre la percepción de apoyo a la autonomía y la satisfacción académica de los estudiantes. Estos modelos permiten probar las hipótesis de mediación de manera eficiente (MacKinnon, 2008).

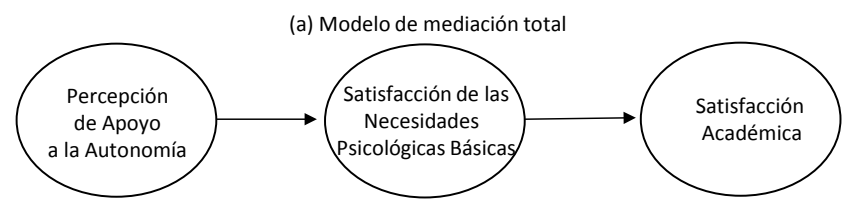

(b) Modelo de mediación parcial

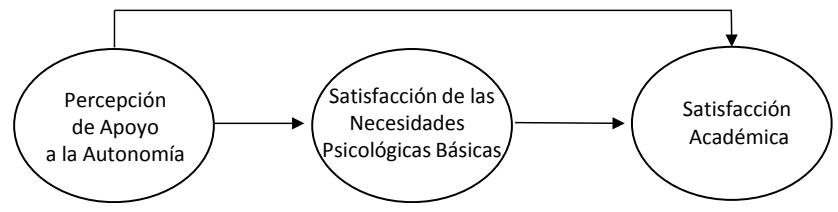

Figura 1. Modelos hipotéticos de ecuación estructural ${ }^{1}$

1 Por simplicidad solo se muestran las variables latentes. 


\section{Resultados}

\section{Resultados descriptivos y correlaciones entre las variables estudiadas}

De acuerdo con los resultados que muestra la tabla 1, las puntuaciones medias aportadas por los estudiantes en cada una de las dimensiones evaluadas son razonablemente elevadas puesto que, en una escala entre 1 y 5 , se encuentran entre $=3.90$ para la percepción de apoyo a la autonomía $\mathrm{y}=4.20$ la competencia.

Tabla 1

Medias, desviaciones típicas y correlaciones entre las variables estudiadas

\begin{tabular}{lccccc}
\hline Variables & Apoyo Aut. & Auton. & Compet. & Relación & Sat.Acad. \\
\hline Apoyo Autonomía & 1 & $.48^{* *}$ & $.52^{* *}$ & $.59^{* *}$ & $.69^{* *}$ \\
Autonomía & & 1 & $.63^{* *}$ & $.60^{* *}$ & $.47^{* *}$ \\
Competencia & & 1 & $.65^{* *}$ & $.48^{* *}$ \\
Relación & & & 1 & $.55^{* *}$ \\
Satisfac. Académica & & & & & 1 \\
$\bar{X}$ & 3.90 & 4.07 & 4.20 & 4.17 & 4.11 \\
$D T$ & 0.70 & 0.63 & 0.56 & 0.59 & 0.70 \\
\hline
\end{tabular}

Nota. ${ }^{*} p<.05 ;{ }^{* *} p<.01$

La dispersión de las valoraciones es moderadamente baja, mostrando desviaciones típicas que oscilan entre 0.56 y 0.70 . Las correlaciones bivariadas muestran que la percepción de apoyo a la autonomía por los profesores se relaciona de manera positiva y estadísticamente significativa tanto con los factores de las necesidades psicológicas básicas (autonomía, competencia, relación) como con la satisfacción académica de los estudiantes. A su vez, las relaciones entre los tres componentes de las necesidades psicológicas básicas también son estadísticamente significativas (entre $\mathrm{r}=.47$ y r =.65, $p<.01$ ).

\section{Predicción de la satisfacción académica}

Para predecir la satisfacción académica de los estudiantes universitarios, se comenzó por poner a prueba el modelo de mediación total que muestra la figura 1, obteniendo los siguientes índices de ajuste: $\mathrm{c} 2(297)=1648.72, p<.001, \mathrm{CFI}=.901$, RMSEA $=.077$ [90\% CI.074 -.081]. El segundo modelo, con mediación parcial, ofrece unos índices de ajuste mejores que el anterior: $\mathrm{c} 2(296)=1117.41, p<.001, \mathrm{CFI}=.940, \mathrm{RMSEA}=.061$ [90\% CI.057 -.064], y por tanto es el que se conserva. 


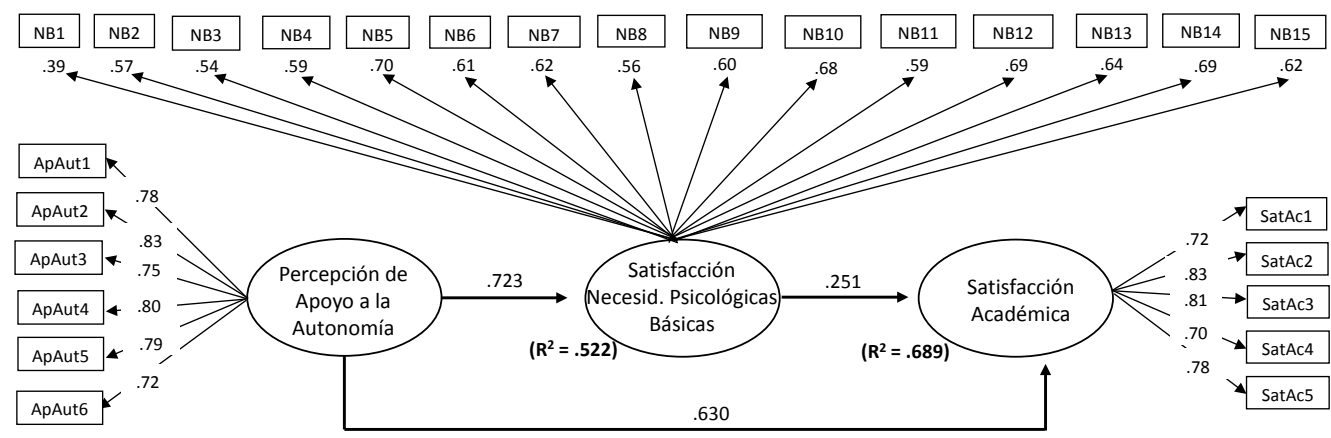

Figura 2. Solución estandarizada para el modelo final probado de mediación parcial@²

Como puede observarse en la figura 2, las saturaciones de los indicadores del apoyo a la autonomía, las necesidades psicológicas básicas y la satisfacción académica resultan elevadas. Excepto el primer indicador de las necesidades básicas (.39), el resto de los indicadores oscilan entre.54 y.83.

La figura 2, que recoge los resultados del modelo estructural con mediación parcial, muestra que la percepción de apoyo a la autonomía por los profesores presenta un efecto positivo sobre la satisfacción de las necesidades básicas de los estudiantes ( $\beta$ $=.723, p<.01)$ y que estas necesidades psicológicas básicas se encuentran positivamente relacionadas con la satisfacción académica de los estudiantes universitarios $(\beta=.251, p$ $<.01)$. Además, también se observa el efecto positivo y directo del apoyo a la autonomía por los profesores sobre la satisfacción académica de los estudiantes $(\beta=.630, p<.01)$. Con respecto a la capacidad predictiva de cada una de las variables, encontramos, por un lado, que la percepción de apoyo a la autonomía predice el 52.2\% de la variabilidad de la satisfacción de las necesidades psicológicas básicas, y por otro, que entre el apoyo a la autonomía y la satisfacción de las necesidades básicas predicen el $68.9 \%$ de la satisfacción académica de los estudiantes universitarios.

\section{Discusión y conclusiones}

Existe evidencia empírica de que el abandono de los estudios por parte de un elevado número de estudiantes universitarios es un problema que preocupa a las autoridades educativas de todos los países y a la sociedad en general (Arce et al., 2015; Freixa, Llanes \& Venceslao, 2018; Magen-Nagar \& Shachar, 2017; Valdés-Henao, 2018). Tal como señalan Feixas, Rodríguez, Muñoz, Navarro y Gairín (2015, p. 118), "reducir la tasa de abandono universitario precisa de un profundo conocimiento del fenómeno y de las causas que lo producen, tanto en el ámbito personal como en el institucional, educativo, social y económico". De aquí que un objetivo actual importante de los psicólogos educativos y de los profesionales de la educación sea analizar qué variables se encuentran relacionadas con tal fenómeno y aportar soluciones al mismo. En este sen-

2 ApAut: apoyo a la autonomía, NB: necesidades básicas, SatAc: satisfacción académica. Todas las relaciones mostradas son significativas con $p<.01$. 
tido, la literatura científica destaca que la satisfacción de los estudiantes con el entorno educativo es un predictor válido de la intención de abandonar los estudios (Suhlmann et al., 2018), y que la satisfacción académica de los estudiantes es un componente de su bienestar subjetivo (Baker et al., 2003; Lent et al., 2007; Seligman \& Csikszentmihalvi, 2000; Wach et al., 2016). Esto se puede ver significativamente afectado por el apoyo a la autonomía prestado por los profesores y por la satisfacción de las necesidades psicológicas básicas de los estudiantes (Gillet et al., 2017; Orsini et al., 2018; Sulea et al., 2015). Por todo ello, encuadrado en el marco de la teoría de la autodeterminación y de la psicología positiva, el objetivo de este trabajo ha sido analizar el carácter predictivo del clima motivacional en el aula (apoyo a la autonomía por los profesores) sobre la satisfacción académica de los estudiantes, actuando como mediadora la satisfacción de las necesidades psicológicas básicas.

A la vista de los resultados obtenidos, la primera hipótesis, que plantea una relación positiva entre el apoyo a la autonomía por los profesores y la satisfacción de las necesidades psicológicas básicas de los estudiantes, se ha visto cumplida. Este resultado se encuentra en la línea de los hallados en investigaciones como la de Liu y Chung (2016) que concluyen la existencia de una relación significativa entre el apoyo a la autonomía por los profesores y la satisfacción de las necesidades básicas de los alumnos en las clases de educación física. También concuerdan con los de Diseth, Breidablik y Meland (2018) que encuentran una relación positiva entre el apoyo a la autonomía por los profesores y la satisfacción de las necesidades básicas de los estudiantes. Cabe matizar que en este trabajo de Diseth et al. (2018) las relaciones encontradas entre el apoyo a la autonomía y la satisfacción de las necesidades son recíprocas.

La segunda hipótesis plantea que la satisfacción de las necesidades psicológicas básicas está positivamente relacionada con la satisfacción académica de los estudiantes. Esta hipótesis también se ha cumplido, y viene a corroborar los resultados de investigaciones previas como las de Evans y Bonneville-Roussy (2016) o Milyavskaya y Koestner (2011). Por su parte, Sulea et al. (2015) terminan concluyendo que si bien ciertos rasgos de personalidad desempeñan un papel en la predicción del bienestar, la satisfacción de las necesidades básicas supone una importancia adicional en relación con el bienestar subjetivo de los estudiantes. Otro trabajo con el que también presenta afinidad el aquí desarrollado es el de Cordeiro et al. (2016) que encuentran un efecto significativo de la satisfacción de las necesidades básicas sobre el bienestar de los estudiantes.

La tercera hipótesis plantea que el apoyo a la autonomía por los profesores está positivamente relacionado con la satisfacción académica de los estudiantes universitarios. Nuestros resultados confirman esta hipótesis y se encuentran en la línea de los obtenidos por autores como López-Walle et al. (2012), Lent et al. (2007) y Griffin (2016), los primeros en contexto físico-deportivo y los demás en otros contextos académicos. En todos los casos el apoyo a la autonomía por los profesores y los entrenadores muestra un efecto positivo sobre el bienestar y la satisfacción de los estudiantes y deportistas. En el estudio de López-Walle et al. (2012) se pone a prueba un modelo estructural similar al que hemos propuesto en nuestro trabajo, y sus resultados evidencian que el apoyo a la autonomía por los entrenadores predice diversos indicadores del bienestar psicológico de los deportistas, entre los que se encuentra la satisfacción con la vida. Además, estos autores llegan a la conclusión de 
que esas relaciones tienen lugar a través de la mediación de las necesidades psicológicas básicas. La diferencia fundamental entre el trabajo de López-Walle et al. (2012) y el que nosotros aquí presentamos, radica en la muestra de estudio (deportistas vs. estudiantes universitarios), y en los indicadores del bienestar (satisfacción con la vida vs. satisfacción académica). En cuanto al trabajo de Lent et al. (2007), estos autores también encuentran un efecto directo de los recursos y apoyos ambientales sobre la satisfacción académica de los estudiantes de ingeniería, y un efecto indirecto entre estas variables mediado por la autoeficacia y las expectativas de resultado. Así pues, el paralelismo entre el trabajo el de Lent et al. (2007) y el nuestro puede decirse que es significativo, a pesar que no haber relacionado exactamente las mismas variables en el modelo sometido a verificación.

En conclusión, nuestros resultados aportan evidencia de que el apoyo a la autonomía afecta positivamente a la satisfacción de las necesidades básicas, que éstas afectan positivamente a la satisfacción académica, y que además encontramos un efecto directo y positivo del apoyo a la autonomía sobre la satisfacción académica de los estudiantes universitarios. Así pues, se cumple el modelo de mediación parcial planteado hipotéticamente en la figura 1.

Cabe señalar que la literatura especializada muestra pocos trabajos en los que se haya buscado explícitamente predecir la satisfacción académica de los estudiantes, y menos aún si se trata de estudiantes universitarios. Por tanto, parece justificado que se siga investigando en este sentido, por un lado para darle más significado a la satisfacción académica de los estudiantes como parte de su bienestar subjetivo, en línea con el planteamiento de la psicología positiva, y por otro, por los efectos que una satisfacción elevada con el centro educativo puede aportar en la disminución de intenciones de abandonar los estudios. Como concluyen Alivernini y Lucidi (2011), el nivel de motivación autodeterminada en los estudiantes está directamente relacionado con la percepción del apoyo a la autonomía por los docentes, y es el mejor predictor de la intención de abandonar los estudios. Abundando en este sentido, Niemiec y Ryan (2009) señalan que el apoyo de los profesores a las necesidades psicológicas básicas de autonomía, competencia y relación, facilita la autorregulación autónoma de los estudiantes para el aprendizaje, el rendimiento académico y el bienestar personal.

A pesar de sus fortalezas, este trabajo también presenta sus limitaciones, por lo que los resultados aquí obtenidos deben interpretarse con cautela. Así, la selección de los participantes ha sido incidental o por conveniencia, lo cual no invalida la verificación del modelo estructural puesto a prueba, aunque sí sería deseable, en un futuro, seleccionar una muestra con mayor garantía de representatividad de la población universitaria dominicana. Por otro lado, el diseño de investigación aquí planteado es un diseño transversal. Es posible que un diseño longitudinal aporte una explicación más clara del comportamiento de las variables relacionadas en el modelo hipotético. Además, todos los datos analizados se han obtenido mediante autoinforme, quedando fuera de nuestro control la posible subjetividad de la respuesta de los participantes. En un futuro, podrían combinarse medidas de autoinforme con medidas más objetivas. A esto, podría añadirse un diseño metodológico mixto en el que se contemplen tanto tratamientos cuantitativos como análisis cualitativos. Finalmente, hay que reconocer que el modelo hipotético aquí planteado, aunque válido, no tiene por qué ser el único, 
pudiendo proponerse otros modelos que aborden este mismo objeto de estudio con diferentes puntos de vista, por ejemplo, teniendo en cuenta que la percepción de apoyo a la autonomía y la satisfacción de las necesidades básicas, pueden actuar como antecedente y como consecuente una de otra, por su posible relación recíproca. Esto mismo podría plantearse para la relación entre el apoyo a la autonomía por los profesores y la satisfacción académica de los estudiantes universitarios. En todo caso, lo que queda claro es que hay que seguir investigando sobre las diferentes variables que pueden estar presentes en el contexto universitario, susceptibles de afectar tanto al comportamiento de los profesores como al rendimiento, satisfacción y bienestar de los estudiantes.

Desde una perspectiva aplicada, la teoría de la autodeterminación puede hacer grandes aportaciones tanto para la práctica como para las políticas de reforma educativa. La incorporación de actividades que implican apoyo a la autonomía puede conducir a una mejor percepción sobre la instrucción en el aula y favorecer la motivación, el aprendizaje y el bienestar subjetivo. Niemiec y Ryan (2009) proponen algunas estrategias para mejorar la autonomía, la competencia y la relación de los estudiantes: (1) las estrategias para mejorar la autonomía incluyen proporcionar opciones y fundamentos significativos para las actividades de aprendizaje, reconociendo los sentimientos de los estudiantes sobre esos temas, y minimizando la presión y el control; (2) las estrategias para mejorar la competencia incluyen proporcionar tareas de evaluación pertinentes a la eficacia, a diferencia de las basadas en normas, de retroalimentación y de desafío óptimo; y (3) las estrategias para mejorar la relación incluyen transmitir calidez, cuidado y respeto a los estudiantes. Finalmente, como señala Griffin (2016), los resultados de trabajos como los aquí revisados deberían ser alentadores para los educadores porque indican que los estudiantes reconocen las conductas de los profesores diseñadas para fomentar un ambiente de aprendizaje positivo, y que cuando incorporan comportamientos de apoyo a la autonomía en una clase universitaria, los estudiantes mejoran la satisfacción de sus necesidades psicológicas básicas, obtienen calificaciones más altas y sienten mayor satisfacción con sus estudios y con su vida en general.

\section{Referencias}

Alivernini, F., \& Lucidi, F. (2011). Relationship between social context, self-efficacy, motivation, academic achievement, and intention to drop out of high school: A longitudinal study. The Journal of Educational Research, 104(4), 241-252. doi: https://doi. org/10.1080/00220671003728062

Arce, M. E., Crespo, B., \& Míguez-Álvarez, C. (2015). Higher education drop-out in Spain. Particular case of universities in Galicia. International Education Studies, 8(5), 247-264. doi: http://dx.doi.org/10.5539/ies.v8n5p247

Baker, J. E., Dilly, L. J., Aupperlee, J. L., \& Patil, S. A. (2003). The developmental context of school satisfaction: Schools as psychologically healthy environments. School Psychology Quarterly, 18(2), 206-221. doi: https://doi.org/10.1521/scpq.18.2.206.21861

Chen, C. (2015). Incremental validity of achievement goals in predicting subjective wellbeing among university students. Journal of Cognitive Education and Psychology, 14(1), 38-62. doi: https://doi.org/10.1891/1945-8959.14.1.38 
Corcino, P. (24 de abril de 2017). Aumenta la deserción universitaria en los jóvenes dominicanos. Periódico El Dinero. Recuperado de https://www.eldinero.com.do/40308/ aumenta-la-desercion-universitaria-en-los-jovenes-dominicanos/

Cordeiro, P., Paixão, P., Lens, W., Lacante, M., \& Sheldon, K. (2016). Factor structure and dimensionality of the balanced measure of psychological needs among Portuguese high school students. Relations to well-being and ill-being. Learning and Individual Differences, 47, 51-60. doi: http://dx.doi.org/10.1016/j.lindif.2015.12.010

Deci, E. L., \& Ryan, R. M. (2008). Self-determination theory: A macrotheory of human motivation, development, and health. Canadian Psychology, 49(3), 182-185. http://dx.doi. org/10.1037/a0012801

Diseth, A., Breidablik, H. J., \& Meland, E. (2018). Longitudinal relations between perceived autonomy support and basic need satisfaction in two student cohorts. Educational Psychology, 38(1), 99-115. doi: https://doi.org/10.1080/01443410.2017.1356448

Evans, P., \& Bonneville-Roussy, A. (2016). Self-determined motivation for practice in university music students. Psychology of Music, 44(5) 1095-1110. doi: https://doi. org/10.1177/0305735615610926

Feixas, M., Rodríguez, D., Muñoz, J. L., Navarro, M., \& Gairín, J. (2015). Hacia la comprensión del abandono universitario en Catalunya: el caso de la Universitat Autònoma de Barcelona. Estudios sobre Educación, 28, 117-138. doi: https://doi.org/10.15581/004.28.117138

Finney, S. J., \& DiStefano, C. (2006). Non-normal and categorical data in SEM. En G. R. Hancock y R. O. Mueller (Eds.), Structural equation modeling: A second course (pp. 269314). Greenwich, CO, EE.UU.: Information Age Publishing.

Freixa, M., Llanes, J., \& Venceslao, M. (2018). El abandono en el recorrido formativo del estudiante de ADE de la Universidad de Barcelona. Revista de Investigación Educativa, 36(1), 185-202. doi: https://doi.org/10.6018/rie.36.1.278971

Gillet, N., Morin, A. J., Huyghebaert, T., Burgger, L., Maillot, A., Poulin, A., \& Tricard, E. (2017). University students' need satisfaction trajectories: A growth mixture analysis. Learning and Instruction. doi: https://doi.org/10.1016/j.learninstruc.2017.11.003

Griffin, B. W. (2016). Perceived autonomy support, intrinsic motivation, and student ratings of instruction. Studies in Educational Evaluation, 51, 116-125. doi: https://doi. org/10.1016/j.stueduc.2016.10.007

Hagger, M. S., Chatzisarantis, N. L., Hein, V., Pihu, M., Soós, I., \& Karsai, I. (2007). The perceived autonomy support scale for exercise settings (PASSES): Development, validity and cross-cultural invariance in young people. Psychology of Sport and Exercise, 8, 632-653. doi: https://doi.org/10.1016/j.psychsport.2006.09.001

Huebner, E. S., Ash, C., \& Laughlin, J. E. (2001). Life experiences, locus of control, and school satisfaction in adolescence. Social Indicators Research, 55, 167-183. doi: https:// doi.org/10.1023/A:1010939912548

Huo, Y., \& Kong, F. (2014). Moderating effects of gender and loneliness on the relationship between self-esteem and life satisfaction in Chinese university students. Social Indicators Research, 118(1), 305-314. doi: https://doi.org/10.1007/s11205-013-0404-x

Jang, H., Kim, E. U., \& Reeve, J. (2012). Longitudinal test of self-determination theory's motivation mediation model in a naturally occurring classroom context. Journal of Educational Psychology, 104(4), 1175-1188. doi: http://dx.doi.org/10.1037/a0028089 
Kline, R. B. (2016). Principles of structural equation modeling. New York, NY, EE.UU.: LEA. Lent, R. W., Singley, D., Sheu, H.B., Schmidt, J. A., \& Schmidt, L. C. (2007). Relation of social-cognitive factors to academic satisfaction in engineering students. Journal of Career Assessment, 15(1), 87-97. doi: https://doi.org/10.1177/1069072706294518

Liu, J. D., \& Chung, P. K. (2016). Students' perceived autonomy support and psychological needs satisfaction in physical education and exercise intrinsic motivation. Journal of Sport Behavior, 39(4), 409-425.

López-Cassá, E., Pérez-Escoda, N., \& Alegre, A. (2018). Competencia emocional, satisfacción en contextos específicos y satisfacción con la vida en la adolescencia. Revista de Investigación Educativa, 36(1), 57-73. doi: https://doi.org/10.6018/rie.36.1.273131

López-Walle, J., Balaguer, I., Castillo, I., \& Tristán, J. (2012). Autonomy support, basic psychological needs and well-being in Mexican athletes. The Spanish Journal of Psychology, 15(3), 1283-1292. doi: https://doi.org/10.5209/rev_SJOP.2012.v15.n3.39414

Ma, C. M., Shenk, D. T., \& Lai, C. C. (2016). Psychological needs, self-regulation, and motivation profiles among a sample of Hong Kong Chinese university students: a person-centered approach. International Journal on Disability and Human Development, 16(4), 407-416. doi: https://doi.org/10.1515/ijdhd-2017-7009

MacKinnon, D. P. (2008). Statistical Mediation Analysis. New York, NY, EE.UU.: Lawrence Erlbaum Associates.

Magen-Nagar, N., \& Shachar, H. (2017). Quality of teaching and dropout risk: A multilevel analysis. Journal of Education for Students Placed at Risk, 22(1), 9-24. doi: https:// doi.org/10.1080/10824669.2016.1242069

Medellín-Lozano, E. W. (2010). Contrastación de dos modelos motivacionales de autodeterminación para predecir la deserción en universitarios. Acta Colombiana de Psicología, 13(2), 57-68.

Milyavskaya, M., \& Koestner, R. (2011). Psychological needs, motivation, and wellbeing: A test of self-determination theory across multiple domains. Personality and Individual Differences, 50, 387-391. doi: https://doi.org/10.1016/j.paid.2010.10.029

Ministerio de Educación, Cultura y Deporte [MECD] (2016). Datos básicos del sistema universitario español. Curso 2015-2016. Madrid: Ministerio de Educación, Cultura y Deporte.

Muthén, L. K., \& Muthén, B. O. (2017). Mplus User's Guide. Eighth Edition. Los Ángeles, CA, EE.UU.: Muthén \& Muthén.

Nearchou, F. A., Stogiannidou, A., \& Kiosseoglou, G. (2014). Adaptation and psychometric evaluation of a resilience measure in Greek elementary school students. Psychology in the Schools, 51(1), 58-71. doi: https://doi.org/10.1002/pits.21732

Niemiec, C. P., \& Ryan, R. M. (2009). Autonomy, competence, and relatedness in the classroom: Applying self-determination theory to educational practice. Theory and Research in Education, 7, 133-144. doi: http://dx.doi.org/10.1177/1477878509104318

Orsini, C., Binnie, V., Wilson, S., \& Villegas, M. J. (2018). Learning climate and feedback as predictors of dental students' self-determined motivation: The mediating role of basic psychological needs satisfaction. European Journal of Dental Education, 22(2), 228-236. doi: https://doi.org/10.1111/eje.12277

Seligman, M. E., \& Csikszentmihalvi, M. (2000). Positive psychology: An introduction. American Psychologist, 55(1), 5-14. doi: http://dx.doi.org/10.1037//0003-066X.55.1.5 
Shih, S. S. (2013). The effects of autonomy support versus psychological control and work engagement versus academic burnout on adolescents' use of avoidance strategies. School Psychology International, 34(3), 330-347. doi: https://doi. org/10.1177/0143034312466423

Suhlmann, M., Sassenberg, K., Nagengast, B., \& Trautwein, U. (2018). Belonging mediates effects of student-university fit on well-being, motivation, and dropout intention. Social Psychology, 49(1), 16-28. doi: https://doi.org/10.1027/1864-9335/a000325

Sulea, C., van Beek, I., Sarbescu, P., Virga, D., \& Schaufeli, W. B. (2015). Engagement, boredom, and burnout among students: Basic need satisfaction matters more than personality traits. Learning and Individual Differences, 42, 132-138. doi: https://doi. org/10.1016/j.lindif.2015.08.018

Tian, L., Han, M., \& Huebner, E. S. (2014). Preliminary development of the Adolescent Students' Basic Psychological Needs at School Scale. Journal of Adolescence, 37, 257267. doi: https://doi.org/10.1016/j.adolescence.2014.01.005

UNESCO (1998). Higher Education Staff Development: Continuing Mission. Recuperado de http://www.unesco.org/education/educprog/wche/principal/mission.html

Urquijo, I., \& Extremera, N. (2017). Satisfacción académica en la universidad: relaciones entre inteligencia emocional y engagement académico. Electronic Journal of Research in Educational Psychology, 15(3), 553-573. doi: https://doi.org/10.14204/ejrep.43.16064

Valdés-Henao, C. (2018). "Deserción universitaria": entre desvinculaciones institucionales y búsqueda de sentidos de vida. Revista Latinoamericana de Ciencias Sociales, Niñez y Juventud, 16(1), 331-344. doi: https://doi.org/10.11600/1692715x.16120

Wach, F. S., Karbach, J., Ruffing, S., Brünken, R., \& Spinath, F. M. (2016). University students' satisfaction with their academic studies: Personality and motivation matter. Frontiers in Psychology, 7(55), 1-12. doi: https://doi.org/10.3389/fpsyg.2016.00055

Wu, A. M., Lai, M. A., \& Chan, I. T. (2014). Coaching behaviors, satisfaction of needs, and intrinsic motivation among Chinese university athletes. Journal of Applied Sport Psychology, 26, 334-348. doi: https://doi.org/10.1080/10413200.2014.888107

Fecha de recepción: 16 de abril de 2018.

Fecha de revisión: 7 de mayo de 2018.

Fecha de aceptación: 16 de noviembre de 2018. 
